\title{
LES CATALANS À DUBROVNIK ET DANS LE BASSIN ADRIATIQUE À LA FIN DU MOYEN ÂGE
}

\author{
NENAD FejIC \\ Université de Poitiers (France)
}

Le commerce international engageait une multitude de Catalans a Dubrovnik et dans le bassin adriatique en général, à la fin du Moyen Age. Il s'agissait de membres des grandes familles commerçantes originaires de Barcelone ou de Tortose, représentés sur les marchés locaux par des procureurs, ou bien d'individus qui tentaient, eux-mêmes leur chance et se lançaient dans les affaires périlleuses à Venise, Dubrovnik ou Ancône. Il y avait aussi, parfois de simples artisans, tisserands, pour la plupart, qui engageaient leur savoir faire et leur labeur, et souvent quittaient leur ville d'adoption ruinés.

Si l'on cherchait à établir, entre la fin du XIV et le début du XVI ${ }^{e}$ siècle, les limites chronologiques de certaines activités des Catalans à Dubrovnik, la fin de la deuxième décennie du $\mathrm{XV}^{\circ}$ siècle (vers 1420), pourrait être considérée comme un tournant. Jusqu' a cette date, l'activité principale des gens originaires de la Péninsule Ibérique à Dubrovnik, ainsi que sur d'autres marchés adriatiques, était la traite des esclaves, ainsi que le commerce du crédit. Par certains de ses aspects, le commerce des esclaves présageait les traits caractéristiques de la technique du commerce de la laine, qui devait atteindre son plein essor au $\mathrm{XV}^{\circ}$ siecle. En effet, la traite des esclaves, fixe pour la première fois un certain nombre de Catalans a Dubrovnik, et contribue à l'élaboration d'un véritable réseau d'affaires entre les Catalans à Dubrovnik et ceux, réstés dans leur pays d' origine. Le circuit du commerce des esclaves, auquel prennent part les Catalans, à travers la Sicile et la Marche (Ancône), correspond à peu près completement au circuit plus tardif du commerce de la laine.

"Anuario de Estudios Medievales". 24 (1994) 
Cependant, tandis que le commerce des esclaves est lié à une brève période de conjoncture dans la Péninsule Ibérique, vers la fin du XIVe siècle, le commerce de la laine amena à Dubrovnik des dizaines, voire des centaines de voyageurs de Catalogne, et favorisa le développement dans la ville de nombreuses activités accessoires, liées à l'industrie textile ${ }^{1}$. Les grands hommes d'affaires qui passent à Dubrovnik plus d'une décennie, tel le consul Jean Exparter (Sparterius), 1439-1469, donnent une image de la communauté catalane, mais dans leur orbite s'affairent, moins visibles, les patrons des navires qui livrent la laine, les marins qui protestent à cause des salaires payés en retard, les petits marchands qui s'interessent à d'autres articles demandés sur le marché catalan (le corail, le bois, l'argent, le plomb), ou bien sur le marché adriatique et plus spécialement ragusain, les peaux, le liège, le sucre, la résine). On y trouve les représentants de diverses activites tertiaires, des notaires, des chirurgiens, des maitres d' écoles, portés par la vague de conjoncture, ensuite des consuls, nommés par les conseillers de Barcelone, pour les aider et les soutenir dans leurs activités, et intervenir dans les nombreux litiges. Les sources concernant les Catalans, ne se résument donc plus à une série d'actes d'achat ou de vente, émanant de leur activité principale: ces sources nous permettent souvent d' entrer dans leur vie quotidienne, d'étudier plus en détail leurs rapports avec la population et les autorités locales ${ }^{2}$.

La vie des représentants les plus en vue de la communauté catalane a Dubrovnik, peut être suivie pendant plusieurs années, voire décennies, grâce surtout à la participation au commerce de la laine. Nous arrivons à reconstruire ainsi le cercle de leur famille et de leurs amis a Dubrovnik, et en Catalogne. Le commerce de la laine et l'industrie textile supposaient une répartition du travail qui avait son incidence sur la vie materielle et la situation sociale des Catalans à Dubrovnik. Les plus grands commerçants catalans, comme nous venons de voir, étaient représentés sur le marché local par des procureurs. Prenons l'exemple de la famille de Roda et de la famille Ghibert. Les sources ragusaines mentionnent trois membres de la célèbre famille de Roda, dont deux séjournèrent sans aucun doute à Dubrovnik. Michel de Roda, grand marchand barcelonais. A la fin de la

\footnotetext{
'Jaime VICENS VIVES, Manual de historia economica de Espana, con la colaboracion de Jorge Nadal Oller, Barcelona, 1967, pp. 196-198.

${ }^{2}$ Il faut surtout we reférer aux nombreux registres de la cour civile ragusain "Sontentiace Cancellariae".
} 
seconde et au début de la troisième décennie du XVe siècle, il passa avec les patrons des navires de nombreux contrats d'affretements pour le transport de grandes quantités de laine à Dubrovnik et à Venise. Si l'on se réfere aux documents ragusains, il se rendit à plusieurs reprises à Dubrovnik, dans l'intervalle de dix ans, de 1419 à 1429.

Les conseils de la ville de Dubrovnik lui octroyerent dans cet intervalle à plusieurs reprises des sauf-conduits pour le séjour dans la ville. Même pendant son séjour à Venise, il prétait de l'argent à certains marchands ragusains, et a Barcelone, il nomma Jean Exparter, le futur consul catalan à Dubrovnik, comme son procureur dans cette ville ${ }^{3}$. Avec son fils Jean, Michel de Roda, en tant que fermier de la "douane italienne", lésa gravement en 1444 trois marchands ragusains qui se trouvaient à Valence, dont le célebre écrivain Benedetto Cotrugli (auteur du traité sur le commerce et le parfait commerçant). Les sources concernant ce conflit se trouvent aussi bien dans les archives barcelonaises que dans les archives ragusaines. En signe de représailles, les Ragusains sequestrèrent ses biens a Dubrovnik, entre les mains du consul Jean Exparter. Michel de Roda mourut, probablement en Catalogne, avant 1454, car dans un document de procuration rédigé la même année à Dubrovnik, son fils Jean de Roda est mentionné comm "filius quondam Michaelis de Roda". Les fils de Michel, Melchior et Jean de Roda, continuèrenet ses affaires dans l'espace adriatique; Melchior réussit à établir, avec un autre marchand catalan Pierre Zordano, à la fin de la seconde décennie du XVe siècle, une société pour le commerce de la laine qui fut représentée à Dubrovnik, par son propre frère, Jean de Roda. Mais, au début des années trente du XVe siecle, il avait déjà abandonné le marché adriatique, et son nom n'etait plus mentionné dans les sources ragusaines. Son frère, Jean de Roda, se rendit aussi à Dubrovnik. Pour la première fois en 1420 , en tant que procureur de son frère Melchior, chargé de vendre la laine a Dubrovnik. Les Ragusains payaient la laine achetée à Jean de Roda, par des lettres de change envoyées à Venise. Plus de deux décennies plus tard, en 1444, Jean de Roda, porta avec

\footnotetext{
${ }^{3}$ A. DE CAPMANY Y DE MONPALAU, Memorias históricas sobre la marina, comercio y artes de la antigua ciudad de Barcelona. Reedición anotada, vol. Il-1, Bancelona, 1962, p. 325.

"Voir, par example, Archivo de la Corona de Aragón, Bailía general, Inventario de procesos antiguos, fasc. 1445-1448; C. CARRERE, Barcelona 1380-1462. Un censre econdmic en zpoca de crisi, Barcelona, 1978, I, p. 340; II, pp. 27, 127.
} 
son père Michel, un grave préjudice aux affaires des Ragusains à Valence, en leur faisant payer injustement la "dohana ytalica" de 200 ducats, à laquelle ceux-ci n'etaient pas ordinairement astreints, en tant que "slaves et non en tant qu'italiens". Cette mesure du père et du fils, marqua le début d'un long conflit, qui engagea, outre les parties interessées, les autorités ragusaines et barcelonaises, la reine Marie d'Aragon, et le lieutenant royal en Catalogne, Galceran de Requesens. En juillet 1445, les áutorités ragusaines confisquèrent les biens de Michel et de Jean de Roda, qui se trouvaient chez Jean Exparter. Une année plus tard, en 1446, le recteur et le Sénat ragusain écrivèrent aux conseillers barcelonais pour se plaindre des mesures illégales de Jean de Roda, qui portaient préjudice aux marchands ragusains. Jean de Roda visita de nouveau Dubrovnik en 1452, et le Sénat invita les marchands lésés à évaluer en commun avec Jean, les dommages subis par sa faute à Valence. Les autorités ragusaines lui demandaient énérgiquement de se présenter devant le tribunal en Catalogne, et de dédommager les marchands ragusains. Jean de Roda promit aux Ragusains qu'il partirait en Catalogne "ubi per libros ipsos omnia videri possent", mais au lieu de donner suite à sa promesse, il partit pour Rome et Naples. Le Sénat ragusain remit alors le dossier directement au roi Alphonse qui décida de lever le sequestre sur les biens de Jean de Roda. A partir de septembre, Jean de Roda ne séjourna plus à Dubrovnik, où il continua à être représenté par le consul Jean Exparter.

Le second exemple d'une grande famille commerçante barcelonaise, très présente a Dubrovnik est celui des frères Ghibert, Berenger, Pierre et Valentin. Berenger, que nous connaissons le mieux, séjourna à plusieurs reprises a Dubrovnik, vers la fin de la troisième et le début de la quatrième décennie du XV siecle. Les frères Ghibert font partie de cette minorité de grands marchands catalans, dont témoignent à la fois les sources ragusaines et barcelonaises. Si l'on juge d'après les très importantes quantités de laine qu'ils assuraient sur le trajet entre Barcelone et Dubrovnik ou Venise (sur un seul navire, ils assurent en 1429, à Barcelone, plus de 20 tonnes de marchandises), et d'après le nombre de sauf-conduits qui leur furent accordés à Dubrovnik, au cours de ces annéess-là, on peut conclure, qu'avec la société de Michel de Roda, ils eurent le premier rôle dans l'exportation de la laine catalane, sur les marchés adriatiques $\mathrm{A}$ la difference de la plupart des sauf-conduits, octroyés aux marchands catalans qui se trouvaient déjà à l'entrée du port de Dubrovnik, dans les sources ragusaines, il est expressément mentionné pour les frères Ghibert "quod intendunt mittere Ragusium 
lanam, granum et alias merces, et hic alia trafica fieri per se et factores suos". La mention des procureurs des marchands catalans a Dubrovnik, ainsi que le fait que le sauf-conduit ne soit pas octroye "ad hoc" montrent que les grands marchands catalans a Barcelone concluaient des contrats pour de très grandes quantités de laine, et qu'entre les deux marchés de laine, il existait un lien continu, qui permettait l'écoulement régulier de la laine catalane sur le marché ragusain.

La laine d'origine catalane commença à affluer à Dubrovnik, ainsi qu'aux deux autres grands marchés adriatiques, Venise et Ancône, avec un retard de vingt années approximativement par rapport aux marchés ligures (Gênes, Livourne, Porto Pisano), et toscans (Prato, Florence). Jusqu'au milieu du XIV ${ }^{\circ}$ siècle, la laine catalane était plutôt exceptionnellement exportée vers les marchés italiens'. Ce ne fut qu'au courant du dernier tiers du XIV ${ }^{\bullet}$ siècle, que les représentants des grandes familles commerçantes florentines, les Medicis, les Pazzi, les Manelli, les Alessandri, les Datini, commencent à exporter régulièrement la laine des centres catalans vers les villes toscanes, foyers d'industrie textile ${ }^{6}$.

Le premier importateur de la laine catalane à Dubrovnik, dont nous pouvons suivre les traces dans les archives, fut un certain Pierre Netti, le Catalan (Petrus Netti, Cathelanus) ${ }^{7}$, en 1418.

Il était installé à demeure à Venise, mais il visitait souvent Dubrovnik, où il avait un procureur permenent en la personne du patricien local Jean de Gradi. Lorsqu'un patron de navire, accusa Jean de Gradi (et par son intermédiaire le Catalan Pierre Netti) d'avoir empeché certains marins de rejoindre leur navire a Dubrovnik, le 29 novembre 1418, il reçut la réponse, qu'il ne s'agissait, en effet, pas de marins, mais des artisans de la

\footnotetext{
${ }^{3} \mathrm{~L}$ 'historien Federigo Melis expliquait le considerable accroissement de l'importation de la laine espagnole dans les villes italiennes, par un changement dans la pratique du versement du nolis, en fonction de la categorie des articles, ce qui devait permettre, pour la premizre fois, la circulation de toutes sortes de marchandises, y compris la laine, que les marchands n'avaient pas interêt à exporter jusqu'alors aux marchés éloignés, du fait de son prix trop bas. Voir à ce sujet: F. MEUS, La lana della Spagna mediterranea e della Berberia occidentale nei secoli XIV-XV, "Atti della Prima settimana di studio" (18-24 aprile 1969), Firenze 1974.

"Sur le rôle de la maison "Dutini" dans l'approvisionnement des villes toscanes, par l'intermédiaire des succursales à Barcelone, voir A. SAPOR, Studi di storia economica, I-II, Firenze, 1955, pp. 161 et 177-178.

'Les Archives historiques de Dubrovnik (Historijski arhiv Dubrovnika: plus loin HAD), série Diversa Notariae XII, 281' et série Consilium Maius II, 14'.
} 
laine: "homines de arte lanae, qui venerant recto tramite Ragusium, pro faciendo dictam artem ${ }^{8}$.

Comment était organisée l'exportation de la laine, et d'autres marchandises acheminées de la Catalogne à Dubrovnik et dans d'autres marchés adriatiques? Les marchands catalans et florentins passaient à Barcelone des contrats d'assurance et d'affretement pour le transport des marchandises dans les ports adriatiques. Les patrons appareillaient alors, le plus souvent vers Tortose, le premier port d'exportation de la laine catalane. Les sources ne laissent pas toujours deviner si le marchand lui-meme se trouvait a bord du navire, ou bien s'il se faisait remplacer par un procureur. La responsabilité pour le frêt passait au patron, qui était tenu respecter certains itinérairres, et livrer à temps la laine ou autre marchandise dans le port adriatique. En tant qu'indices pour la quantification de l'exportation de la laine sur les marchés adriatiques, les sources catalanes sont insuffisantes. Des tentatives indirectes ont été faites dans l'historiographie moderne, par le biais de l'évaluation des primes payées lors de la rédaction des contrats d'assurance a Barcelone'. Les résultats de tels efforts ne sont valables que pour des intervalles réduits, à cause de l'état inégal des registres notariaux. Une difficulté supplémentaire est occasionnée par le fait que les plus grands marchands seulement, tels le père et les fils de Roda, pouvaient se permettre d'assurer la totalité de la cargaison, alors que les autres devaient se contenter d'une couverture mineure de la valeur de la marchandise.

$\mathrm{Au}$ cours d'un an et demi, de juillet 1428 à décembre 1429, le notaire barcelonais, Bartholomé Masons, avait rédigé les actes d'assurance de la laine, dans une valeur de 36.000 livres de monnaie barcelonaise ${ }^{10}$. En partant du prix d'une rove de laine, qui coutait sur le marché barcelonais approximativement 20 sous, ou bien une livre de monnaie barcelonaise, l'historien italien Mario Del Treppo a calculé, qu'au cours de la période

'Consilium Maius II, 14'.

'M. DEL TREPPO, Els mercaders catalans il 'expansio de la Corona casalano-aragonesa al segle XV, Barcelona 1976.

${ }^{10} \mathrm{M}$. DEL TREPPO, Els mercaders, p. 240. Pour les problemes monethires de l'epoque, voir aussi, M. BLOCH, Esquisse d'une histoire monétaire de l'Europe, "Cahiers des Annales", 9 (1954), p. 24; C. CIPPOLA, Moneta e civilta mediterranea, Venezia, 1957, surtout les chapitres: I dollari del medioevo (pp. 30-32) et Le monete fantasma (pp. 51-65); R. LOPEZ, Settecento anni fa, il ritomo all' oro nell' occidente duecentesco, "Rivista Storica Italiana", 65-1 (1953), pp. 18, 55; 65-2 (1953), pp. 161, 169; F. MATEU Y LLOP1S, Glosario Hispónico de Numismática, Barcelona 1946, p. 206. 
envisagée seulement (1428-1429), Bartolomé Masons avait enregistré l'exportation de 9.000 quintaux, c'est à dire de plus de 360 tonnes de laine vers Gênes, Pise, Venise et Dubrovnik ${ }^{11}$. Le prix de la laine sur le marché catalan était exprimé en une monnaie de compte, la livre barcelonaise de 20 sous de deniers ( 1 livre $=20$ sous $=240$ deniers), qui cachait evidemment la monnaie réelle, c'est à dire, le plus souvent, le florin aragonais ou bien le croat d'argent ${ }^{12}$. Au XV siècle, le prix de la laine évoluait généralement autour de 20 sous pour une rove, sauf pendant les années de grande conjoncture, où il bassait à 15 à 17 sous ${ }^{13}$. Malgré les disparités dans les ordres de grandeur (dans les ports catalans on embarquait de tres grandes quantités de laine, dont une partie seulement était destinée à Dubrovnik), les differences de prix sont sensibles ${ }^{14}$. Toujours partant du registre de Bartholomé Masons, Claude Carrère a tenté de quantifier les exportations de laine vers le "Golfe de Venise", et elle est arrivée à la totalité de 11926 livres de poids, enregistrés par ce notaire seulement, ou bien si l'on admettait le prix de 20 sous pour une rove, au poid de 2981 quintaux, c'est a dire plus de 119 tonnes ${ }^{15}$.

Il s'agit, bien entendu, de la quantité minimale, à condition évidemment que la totalité de la marchandise ait été assurée, ce qui arrivait rarement. Une articulation plus poussée du marché adriatique, en marchés vénitien, ragusain, anconitain, etc., à partir des seules sources catalanes, nous parait tout a fait incertaine. Il arrivait, en effet, souvent, que la laine acheminée à Dubrovnik, soit débarquée à Venise, à la demande des expediteurs eux-memes, par le biais de leurs procureurs, dans l'une ou l'autre ville, a la suite d'un changement de conjoncture imprevisible. Dans les rares occasions, où une subdivision du marché adriatique a été possible, les sources mettent en évidence les différences entre les prix d'affrétement et de nolis pour le transport des marchandises vers chacune de ces villes. Bien

\footnotetext{
"M. DEL TREPPO, Els mercaders, p. 241; Quintal: unité do poids de 4 roves. $4 \times 10,4$ $\mathbf{k g}=41,6 \mathrm{~kg}$.

${ }^{12}$ Voir tableau A

${ }^{13}$ M. DEL TREPPO, Els mercaders, p. 240; entro 1430 et 1436, l'exportation de la laine atteint son apogé; 10 prix de la laine a Barcelone était alors a son niveau le plus bes, c'est a dire, 15-17 sous.

${ }^{14}$ Voir les tableaux A et B

${ }^{15} \mathrm{C}$. CARRERR, Barcelone, centre économique a l'époque des difficultés. 13801462, 2 vols., Paris, 1967, II, p. 601.
} 
qu'ils soient insuffisants, les renseignements sur les quantités de laine acheminées vers les ports adriatiques, que nous obtenons des registres d'assurances barcelonais sont les seuls dont nous disposons pour établir une statistique de l'exportation de la laine ibérique vers Dubrovnik ${ }^{16}$.

Malgré une forte participation des sociétés commerciales toscanes implantées a Barcelone (Aldobrandi, Davanzzati, Ventura, Pazzi, Nerone, etc.), dans l'approvisionnement du marché ragusain en laine, les plus grandes quantités furent acheminées des villes catalanes par les hommes d'affaires locaux, par l'intermédiaire de leurs procureurs installés à Dubrovnik. Les rares contrats d'assurance et de nolis, dont la majorité date des années trente du XVe siecle, indiquent que la marchandise était transportée par voie maritime, par le biais des ports siciliens. Dans un certain nombre de contrats, les marchands assurent expresement de nouvelles quantités à Messine, qui y seraient chargées après le débarquement de la marchandise arrivée de Tortose ou de Barcelone. Federigo Melis a relevé, il est vrai, un autre itinéraire possible de la laine espagnole vers les ports adriatiques: la laine pouvait être débarquée dans les ports ligures (porto Pisano, Gênes, Livourne), pour être acheminée par voie terrestre jusqu'à Ravenne ou Ancône, et ensuite embarqué à nouveau, pour être acheminée vers Venise ou Dubrovnik ${ }^{17}$.

Le commerce de la laine espagnole dans les ports adriatiques au $\mathrm{XVe}$ siècle, et surtout pendant la quatrième et cinquième décennie, se développait dans les conditions d'existence d'un marché particulièrement stable, qui pouvait à tout moment absorber toutes les quantités offertes. A cette époque, les autorités

\footnotetext{
${ }^{16}$ Malheureusement, l'exportation annuelle de la laine de 119 tonnes à Dubrovnik, qu'a enregistrée le notaire Bartholomé Masons à Barcelone, ne peut être directement confrontée à aucune valeur sûre, provenant des archives locales ragusaines, faute de documentation parallele de même nature. Le seul document d'interet pour l'historien de l'économie, est celui de l'humaniste ragusain, Philippe de Diversis, d'origine lucquoise, qui dans sa "Description de Dubrovnik", affirnait que la production annuelle de laine dans la ville, à peu pres a la meme époque, était de 4.000 rouleaux de tissus. En partant de ce renseignement, et d'une décision gouvernementale ragusaine, qui fixait le poids d'un rouleau à 75 livres, l'historienne D. DinicKnezevic a conclu que 300.000 livres de laine étaient traitées annuellement à Dubrovnik, ce qui correspond approximativement à $143.000 \mathrm{~kg}$., il s'agit done du mème ordre de grandeur.

${ }^{17}$ F. MELIS, La lana della Spagna mediterranea, p. 247; des preuves directes, illustrant ce second trajet de la laine espagnole, n'existent pas. Il est vrai que les documents prouvant l'arrivée de la laine espagnole de Venise à Dubrovnik, confirment indirectement cette thèse de F. Melis.
} 
ragusaines accordaient souvent les permis de séjour aux Catalans, en les affranchissant des droits de douane, et en leur pardonnant les méfaits de leurs compatriotes pirates, en haute mer.

Etant donné que la commune ragusaine n'achetait pas directement la laine aux Catalans, on ne trouve pas dans les sources locales, outre les permis généraux d'importation, d'autres documents qui permettraient de faire une statistique de l'importation de la laine ${ }^{18}$. Même quand elle tentait de proteger les tisserands locaux de la concurence étrangère, la Commune ragusaine avait rarement recours aux mesures de rigueur, pour la simple raison que l'approvisionnement en laine était rarement entre les mains des hommes d'affaires locaux; sur les douanes usuelles pour la laine importée, appliquées aux marins et marchands catalans, on ne sait pas grand chose non plus, car elles ne sont que rarement évoquées dans les sources. Le pourcentage souvent évoqué dans la littérature historique de $1 \%$, nous parait vraisemblable, car la douane appliquée à Valence, pendant les années trente du XVe siècle, atteignait 3 deniers sur la valeur d'une livre de la marchandise exportée, c'est à dire $1,25 \%{ }^{19}$.

Les autorités ragusaines disposaient quand-même de renseignements assez précis, concernant les livraisons de laine par les marchands et patrons de navires catalans. Après le déchargement, la marchandise était soumise au pesage et au dédouanement, en attendant qu'elle soit revendue au détail aux tisserands ragusains. Les notaires de la douane notaient le poids total de la laine et le nombre de sacs, et le marchand était tenu de soumettre dans les plus bréfs délais, aux officiers de la laine communaux, le procèsverbal du notariat de la douane. En cas de litige, entre l'acheteur et le

\footnotetext{
${ }^{18}$ Les sommes payées à Dubrovnik étaient des nolis et non des remboursements pour la laine livrée, qui étaient effectués à Barcelone par lettre de change ou en liquide. Les livraisons de laine étaient le plus souvent à l'origine des litiges entre les acheteurs et les fournisseurs, patrons des navires. Ces litiges étaient souvent reglés par les arbitres d'accomodement. Cependant, grâce aux registres de la cour civile, nous disposons de renseignements qui témoignent des quantités de laine fournics au marché de Dubrovnik. Ainsi, le patron catalan, Aloy Onis, foumit en une seule fois au procureur d'un grand marchand florentin à Dubrovnik, Enrico Arriguci, 95 miliaires, approximativement $45.000 \mathrm{~kg}$. de laine. Cette quantité peut être mise en rapport avec la quantité de laine assuréc à Barcelone, par le Catalan Michel de Roda en 1430, pour un trajet à Dubrovnik: 4.400 roves $(45.760 \mathrm{~kg})$.

(Voir tableaux A et B).

${ }^{19}$ Il s'agit de la célébre Dohana Ytalica: en septembre 1438, le roi Alphonse d'Aragon ordonna au baile général de Valence de ne plus exiger la douane de 3 deniers pour une livre de marchandise importée. L'interdiction a été renouvelée en 1442.
} 
vendeur à Dubrovnik, les officiers de la laine nommaient des estimateurs qui recevaient, chacun un ducat en contrepartie de leur service.

A l'époque de l'essor de l'industrie textile fut élaborée l'image idéale du Catalan à Dubrovnik, que nous retrouvons le plus souvent dans les sources ragusaines. "Il arrive souvent que les tisserands achetent la laine aux Catalans, pour constater ensuite qu'elle est avariée, brulée ou d'origine africaine (barbaresca), ce qui porte grand préjudice à l'industrie textile", constate une disposition du Grand Conseil de Dubrovnik, en 1432, à propos des activités des officiers de la laine. Vers la fin de la même décennie, une décision prise au Grand Conseil de Dubrovnik, nous fait savoir que certains tisserands dans la ville achetent aux marchands catalans plus de laine qu'ils ne sont capables de transformer en tissus, pour la vendre ensuite à des prix superieurs, portant ainsi prejudice au bon fonctionnement de l'industrie textile à Dubrovnik. Pour empecher cette pratique, le Grand Conseil de Dubrovnik prescrit que la laine livrée à Dubrovnik ne peut être revendue qu'après un délai de quatre jours.

En étudiant les causes d'une présence accrue des Toscans, particulièrement des citoyens de Prato à Dubrovnik, vers les années trente du XV siècle, l'historienne yougoslave, Mirjana Popovic-Radenkovic a constaté d'après le contenu des livres de cadastre florentins, "qu'il s'agissait de gens aux propriétés terriennes tres modestes, et aux sommes d'argent liquide encore plus modestes" 20 . Parmi les gens originaires de Prato et de certaines autres villes toscanes, pas un seul ne possedait un atelier de tisserand dans sa ville natale. Il en va tout autrement si l'on se refere aux documents catalans concernant les marchands qui entretenaient des rapports d'affaires avec les centres portuaires du bassin adriatique. Ainsi, les membres de la famille de Roda, le père Michel, et les fils Melchior et Jean, appartenaient au milieu restreint des plus grands hommes d'affaires de Barcelone et Michel de Roda était membre du Conseil des Cent de cette ville ${ }^{21}$. Les frères Ghibert, Berenger, Valentin et Pierre, sont aussi souvent évoqués dans les sources catalanes, Valentin Ghibert en tant que membre du Conseil des Cent et du Conseil des Cinq en 145322. Guillaume, fils de Clémént de

\footnotetext{
${ }^{20} \mathrm{M}$. POPOVIC, La penetrazione dei mercanti Pratesi a Dubrovnik Ragusa) nella prima meld del XV secolo, "Archivio Storico Italiano", 177 (1959), pp. 503-521.

${ }^{21} \mathrm{C}$. BATLE GALART, La crisis social y economica de Barcelona a mediados del siglo XV, 2 vols., Barcelona, 1973, I, pp. 139-140.

${ }^{2}$ Op. cil., p. 453 a passim.
} 
Tortose, qui séjourna à Dubrovnik en 1445, en tant que procureur de son frère François, était aussi désigné par le roi Alphonse de Naples au poste de consul des Catalans a Venise, mais ce fut Antoine Font qui finalement acceda à ce poste par la volonté des conseillers barcelonais. Les membres des grandes familles catalanes, Castellon, Cassassagia, Clement, Sampol, Daghilar, etc., dont on retrouve les noms dans les sources ragusaines, sont aussi souvent mentionnés dans les documents barcelonais en tant que marchands et chargés de certaines fonctions publiques dans la capitale catalane, le Conseil des Cent, le Conseil des Cinq, etc. Il est aussi vrai, qu'ils choisissaient plus souvent Venise que Dubrovnik pour siège de leurs multiples activités économiques, d'où ils commandaient la laine et l'acheminaient à leurs nombreux procureurs installés à Dubrovnik ${ }^{23}$. Les noms des procureurs des grands marchands barcelonais sont mentionnés à Dubrovnik plus souvent que les noms de leurs chefs, bien qu'ils soient pratiquement inconnus dans la vie publique et politique de Barcelone ${ }^{24}$.

En tant que procureurs de petites et moyennes compagnies familiales, certains Catalans séjournaient pendant de longues années à Dubrovnik, et entretenaient des rapports avec les centres économiques de leur pays natal. A la différence de leurs compatriotes plus célèbres, installés à Barcelone ou à Venise, ils ne se limitaient pas à la livraison de la laine. Avec les tisserands et les marchands locaux ragusains, ils passaient des contrats de commerce de la laine, et participaient plus ou moins à toutes les étapes de sa transformation. Certains ont vécu à Dubrovnik pendant des décennies, et ont considérablement contribue a l'essor général de la ville. Le commerce de la laine leur a ouvert la porte du crédit, où il leur arrivait d'investir de très importantes sommes d'argent.

Les représentants typiques de cette couche moyenne des marchands catalans à Dubrovnik, qui laissèrent des traces profondes dans les archives locales, furent Bernard Guasquill, fils de Raymond Guasquill de Tortose, et les frères Jean et Antoine Brull, fils de Huguet Brull de Tortose. Jean Brull, en tant que représentant de la maison familiale à Tortose, que consti-

\footnotetext{
${ }^{23}$ ll s'agissait souvent des membres cadets des grandes familles barcelonaises. Melchior de Roda a séjoumé plusieurs années à Venise, ou il a établi une société pour le commerce de la laine avec le marchand catalan Pierre Jordan.

${ }^{24}$ Le cas le plus interessant est celui de Jean Exparter, procureur de la maison de Roda à Dubrovnik, a partir de 1439. A Barcelone, par contre, il est connu seulement comme procureur d'un autre célèbre inarchand, Jean de Toralba, dans la ville de Pise.
} 
tuaient son père Huguet, et son frère Antoine, séjourna à Dubrovnik de 1426 à 1436, lorsqu'il retourna à Tortose, où il morut, probablement en 1442. De 1430 a 1435 , Jean Brull vendit aux tisserands ragusains, au moins 120 miliaires de laine, c' est à dire 1396 quintaux selon la mesure catalane. Qu'il s'agit là d'une quantité considérable, on peut en juger, en se référant au renseignement de l'historien italien M. Del Treppo, concernant les 9.000 quintaux de laine exportés sur les marchés de Gênes, de Venise, de Pise et de Dubrovnik, dans l'intervalle 1428-142925. Le profit de la vente de la quantité mentionnée sur le marché de Dubrovnik, a supposer que le prix moyen de la laine sur le marché local ait été de 50 ducats par miliaire, était de l'ordre de 6.000 ducats.

Les patrons des navires qui transportaient la laine à Dubrovnik sont aussi bien connus des sources catalanes: Bonanat Conill, Michel Prats, Laurent Landrich et d'autres moins connus. Bonanat Conill fut certainement le plus célebre parmi eux: à partir de Barcelone, il entreprenait des voyages en Afrique du Nord, en Sicile et dans les ports adriatiques ${ }^{26}$. Son nom est souvent mentionné dans les registres d'assurance du notaire barcelonais Bartolomé Masons, pour les voyages vers Venise et Dubrovnik, et avec les grands hommes d' affaires catalans il concluait des contrats de nolis, pour le transport des marchandises dans le "Golfe de Venise". Les sources ragusaines et barcelonaises laissent entendre que certains marchands et marins catalans reliaient pendant des années des ports d'exportation de la Catalogne avec les marchés de la laine adriatiques en particulier, Dubrovnik, Venise et Ancône.

Les principales formes de participation des Catalans dans le commerce de la laine à Dubrovnik étaient la vente directe et la constitution des sociétés pour la vente ou la transformation de la laine. La forme la plus simple était la vente d'une certaine quantité de laine, au prix fixé par miliaire. Cette forme, assez rare, ne supposait pas nécéssairement la vente à crédit. C'est en même temps la seule qui nous permet de procéder à une certaine quantification du poids et du prix de la laine vendue. La seconde forme était beaucoup plus compliquée, et consistait à crediter l'acheteur pour une quantité de laine, avec obligation de sa part de rembourser sa

\footnotetext{
${ }^{25} \mathrm{M}$. DEL TREPPO, Els mercaders, p. 240.

${ }^{26}$ Voir à son sujet C. CARRtRE, Barcelone, centre économique, pp. 233, 237, 266, 274 , 541, 603, 605, 606, 628, 640; M. DEL TREPPO, Els mercaders, pp. 239, 432, 460, 466, 508, 509.
} 
dette en tissus élaborés. Cette forme sous-entend nécéssairement des données sur la quantité de la laine vendue, les prix, sans omettre le nombre de rouleaux à remettre au vendeur ainsi que les delais précis. Très élaboré, un tel contrat pose néanmoins au chercheur moderne de nombreuses difficultés. L'obligation du vendeur catalan est clairement formulee, et elle consistait a livrer une quantité de laine, exprimée en sacs ou en miliaires. Mais, il n'en va pas de même, avec l'obligation de l'acheteur, qui était généralement tenu de rembourser une partie de sa dette en tissus et l'autre en ducats. Le prix n'était généralement pas détérminé au moment de la rédaction du contrat, et ce qui était seulement précisé, c'était la difference entre le prix qui serait en vigueur a Dubrovnik au moment de la livraison de la laine, et le prix réel que l'acheteur payerait au marchand catalan. Cette différence atteignait cinq ducats par miliaire au maximum ${ }^{27}$.

Le nombre de tissus que l'acheteur devait transformer n'est pas précisé, car il évoluait naturellement en fonction du prix de la laine livrée. De nombreux documents dans les registres de la Cour civile (Sententiae Cancellariae), témoignent que les contrats entre les marchands catalans et les tisserands ragusains n'étaient souvent pas réspectés. Parfois le marchand tardait avec la livraison de la laine, ou bien le tisserand n'honorait pas à temps sa dette en ducats, ou en nombre de tissus qu'il s'était obligé de rendre au vendeur.

Outre dans le commerce de la laine, les Catalans a Dubrovnik participaient dans les sociétés pour la fabrication des tissus. Ils investissaient dans ces sociétés une part de leur laine et de l'argent comptant, et leurs partenaires. les tisserands ragusains ou toscans, contractaient l'obligation de produire des tissus.

Les marchands catalans recevaient de leurs partenaires locaux, le plus souvent des tissus a demi élaborés, qu'ils devaient declarer aux officiers communaux de la laine. Ces tissus étaient ensuite soumis au lavage, à la teinture et au cardage. Les marchands catalans les vendaient ensuite a des prix beaucoup plus favorables. Le profit était partagé dans la société, une fois que les membres avaient récuperé les capitaux investis, le Cata-

\footnotetext{
${ }^{27} \mathrm{~L}$ 'acheteur gardait le droit de ne pas recuperer la laine, si elle serait vendue à un prix superieur à 60 ducats.
} 
lan, dans l'achat et la transformation du tissu, et le Ragusain dans le tissa$\mathrm{ge}^{28}$.

A Dubrovnik, les Catalans entretenaient des rapports avec leurs compatriotes, avec les artisans des villes italiennes et avec les Ragusains. Dans l'ensemble de leurs activités, ils privilégiaient quelques acheteurs et quelques partenaires dans la production des tissus, mais les procès devant la cour civile témoignent d'un certain échéc dans certains partenariats.

Il ne faut pas oublier, enfin, un certain nombre de Catalans qui, en tant que tisserands, ont prété leur travail dans la production des tissus, sans pourtant livrer la laine ou investir des capitaux.

Les Catalans ont commencé a s'interesser à la production par le biais du commerce des tissus. Le commerce leur a fourni des crédits. Les premiers fourniseurs de la laine catalane étaient simplement des marins ou des commerçants. Vers le milieux de la troisième décennie du XVe siècle, les facteurs des grandes familles catalanes s'aventurèrent dans la production des tissus, en y investissant d'importants crédits. Claude Carrère soulignait le rôle décisif du commerce sur l'exemple de la Catalogne. D'après cette historienne, les marchands catalans, même s'il ne dominaient pas la production, controlaient les échanges internationaux, dont dépendait la production des tissus. C'est aux marchands que la production des tissus devait sa naissance, sa maturité, et même sa chute, si proche de la période de splendeur ${ }^{29}$.

De même que nous ignorons la quantité totale de la laine livrée à Dubrovnik par l'intérmédiaire des marchands catalans, nous ignorons les effets commerciaux de leur entreprise. La valeur totale de l'exportation des tissus ragusains en Serbie et en Bosnie, au cours de la premiere moitié du $\mathrm{XVe}$ siècle, selon l'historien Jorjo Tadic, était de 250.000 ducats $^{30}$. Plus recemment, en se basant sur des calculs indirects, l'historienne Dusanka

\footnotetext{
${ }^{28}$ Le marchand catalan $s$ 'obligeait a ne pas vendre la moindre quantité de laine a credit, sans permission du tisserand, et le tisserand ragusain s' obligeait à ne pas mettre son atelier et son savoir faire au service d'une tieree personne. Aucune des deux parties n'avait pas le droit d'emprunter de l'argent pour l'achat ou le traiteınent de la laine au nom de la société commune. Les conditions dans lesquelles la socićté pouvait être dissoute avant son terme legal, étaient aussi énumćrées: la mort du marchand ou du tisserand, la livraison incomplète de la laine, la peste ou la guerre.

${ }^{29} \mathrm{C}$. CARRtire, La draperie en Catalogne et en Aragon au XVe sizcle, "Alti della Seconda Settimana di Studio, Istituto Francesco Datini", Prato, Firenze, 1976.

${ }^{30}$ J. TADIC, Privreda Dubrovnika i srpske zemlje u prvoj polovini XV veka, "Zbomik Filozofskog Fakulteta", 10-1 (1968), pp. 519-536.
} 
Dinic Knezevic a révisé cette valeur à la baisse, pour la stabiliser a 40.000 ducats $^{31}$.

Une bonne partie des capitaux investis en crédits à Dubrovnik, provenait du commerce des tissus, auquel les Catalans se livraient sur d'autres marchés balkaniques et méditerranéens. certains créditeurs parmi les gens de la péninsule Ibérique participaient à la production des tissus, même s'ils étaient arrivés à Dubrovnik en premier lieu comme représentants des grandes familles commerçantes de Barcelone ${ }^{32}$. Certains Catalans participaient a la production des tissus, tout en restant en dehors du commerce à crédit, auquel cette production était liée à Dubrovnik ${ }^{33}$.

Qu'il s'agisse de documents officiels, par lesquels le Conseil de Barcelone nomme les consuls à Dubrovnik, ou des actes privés sur l'assurance et le nolis pour le transport des marchandises vers "le Golfe vénitien", les sources catalanes mettent au premier plan le marchand et le marin. Dans les parties dispositives des actes désignant les consuls catalans a Dubrovnik, on mentionne toujours "les marchands qui commercent plus que d'habitude à Dubrovnik". D'autre part, dans les sources ragusaines, le Catalan n'est pas seulement marchand, mais aussi bien participant actif dans la production des tissus. Les entreprises catalanes a Dubrovnik étaient favorisées par un certain nombre de circonstances favorables: en Catalogne, la persistance de la conjoncture favorable jusqu'au milieu du XVe siecle, et sur les marchés adriatiques et balkaniques, les perspectives du commerce de la laine et d'investissements dans la production des tissus ${ }^{34}$. Bien que les circonstances évoquées aient favorisées les rapports directs entre les villes catalanes et Dubrovnik et d'autres villes adriatiques, jusqu'au début de la septieme decennie du XVe siècle, les événements ultérieurs ont montré que le séjour des marchands catalans dans ces villes était dú principale-

${ }^{31}$ D. DINIC-KNEZ.EVIC, Tkanine u privredi srednjovekovnog Dubrovnika, Belgrade, 1982.

${ }^{32}$ Le meilleur exemple est foumi par le personnage de Jean Exparter (Sparterius), futur consul catalan à Dubrovnik, dont la participation à l'industrie textile n'est, en aucun cas, comparable à ses activités de crédit.

${ }^{33}$ Dans la grande ville catalane, les marchands sont aussi des patrons. Mais les artisans de la laine, en particulier les cardeurs, jouent aussi un rôle important. Ils n'achelent pas la laine et ne la revendent pas sur les marchés étrangers, mais entre cess deux étapes, ils dominent souvent, en partie ou dans sa totalité, la production de la lainc; C. CARRERE, La draperie en Catalogne et en Aragon, p. 486.

${ }^{34}$ P. VIIAR, La Calalogne dans l'Espagne inoderne, I, Paris, 1973, pp. $472-476$. 
ment à la conjoncture dans les pays de la Couronne d'Aragon. L'époque de la prépondérance du patriciat à Barcelone, de la domination des grands marchands, intéressés aux échanges internationaux, et peu favorables au protectionisme, jusqu'en 1453, est en même temps l'époque de la présence majeure des Catalans à Dubrovnik.

L'époque de la domination de la moyenne bourgeoisie, avec le soutien du lieutenant royal, suivie de la guerre dynastique et civile en Catalogne, contre la dynastie aragonaise, fut à l'origine du déperissement presque total des rapports maritimes et commerciaux entre les ports catalans et adriatiques ${ }^{35}$. D'autre part, la consolidation du pouvoir ottoman dans l'arrière pays direct de Dubrovnik, menaçait la liberté même de la vile slave $^{36}$. Pendant un bref intervalle la production des tissus dut etre interrompue. Même lorsque le danger immédiat disparut, lorsque l'industrie du textile commença à se rétablir vers la fin du XV et le début du XVI siècle, la participation des Catalans dans cette industrie diminua sensiblement. Le circuit du commerce et la production de la laine ont été coupés, et les efforts individuels en vue de leur rétablissement, sans pour autant que l'on puisse rétablir les liens avec les grands fournisseurs de la Catalogne, étaient d'avance condamnés à l'échec.

Pendant les années des relations les plus intensives, les documents barcelonais témoignent de la présence d'autres marchandises sur les navires catalans en direction de l'Adriatique. Par rapport à la laine, article principal de l'exportation catalane, ces marchandises étaient peu nombreuses. C'est ainsi que les registres d'assurances de 1442-1443, mentionnent une modeste quantité de peaux tannées, expediées de Barcelone à Dubrovnik et Veni$\mathrm{se}^{37}$. Leonard Benet de Barcelone assura en 1460, pour un voyage de Tortose a Dubrovnik, outre la laine, une quantité de sparte utilisée dans le batiment ${ }^{38}$. Vers la fin du siècle, une importante quantité de laine, de liège, de sucre et de résine fut acheminée de Barcelone à Dubrovnik ${ }^{39}$. Il est

\footnotetext{
${ }^{35} \mathrm{C}$. CARRire, Barcelone, centre économique, pp. 655-918; P. VILAR, La Catalogne dans l'Espagne moderne, chapitre: "Guerre civile et approfondissement de la crise (1462-1472)".

${ }^{36}$ Le gouvemement de Dubrovnik ordonna en 1463 de detruire tous les bâtiments aux abords de la ville, surtout les ateliers des tisserands et les teintureries, et de mettre la pierre a la disposition des defenseurs de la ville.

${ }^{37} \mathrm{C}$. CARRERE, Barcelone, centre économique, II, p. 601.

${ }^{38}$ Esteban Mir, Sexti manualis securitatum, 1460-1461.

${ }^{39}$ Pedro Triter, Manualis securitatum, 1496-1498.
} 
interessant de signaler que les sources ragusaines, outre la laine, ne font pas état d'autres articles de commerce importés de Catalogne.

Nous nous heurtons à de plus grandes difficultés encore, lorsque nous essayons de préciser la nature et la quantité des marchandises qui étaient acheminées de Dubrovnik vers la Catalogne. Si les sources catalanes évoquent la laine comme le seul article d'exportation vers "le Golfe de Venise" (avec quelcuues exceptions sans importance), les sources ragusaines sont tout à fait muettes sur l'exportation des articles locaux vers les ports catalans.

Les surplus réalisés à l'époque de la prospérité des Catalans à Dubrovnik, pouvaient être investis dans les affaires des lettres de change, dans leur ville natale ou au Levant ${ }^{\dagger 0}$. Ces surplus, comme l'a remarqué Claude Carrère, pouvaient aussi être investis dans l'achat des métaux precieux dans les Balkans, en Serbie ou en Bosnie ${ }^{41}$. Cependant, aucune de ces hypothèse n'est pas confirmée par les sources catalanes ${ }^{42}$.

Les sources ragusaines par contre, permettent les deux possibilités, mais ne donnent pas assez d'arguments pour les étayer. Les sources sont particulièrement pauvres en renseignements sur l'exportation des métaux precieux en Catalogne: dans la litterature historique, on a souvent évoqué l'afluence de l'argent dans les ports catalans, au cours du XIVe et XVe siecles $^{43}$. Cependant, les arguments évoqués à l'appui temoignent plutôt de la participation des Catalans dans le commerce de ce métal précieux à Dubrovnik, que de son arrivée certaine dans les ports catalans ${ }^{44}$.

\footnotetext{
${ }^{40}$ Bien qu'il y eut quelques exceptions notoires, les affaires directes, par lettres de change entre Catalans à Dubrovnik et leurs compatriotes à Barcelone ou dans d'autres villes catalanes, étaient dans l'ensemble asscez rares.

${ }^{4 !}$ C. CARRERE, Barcelone, centre économique, II, p. 604 "...Métaux précieux de Bosnie, en barres ou monnayés, dueats vénitiens, representent certainement une contrepartic recherchée aux ventes de laine, soit qu'on les utilise sous fonne d'espèces, soit qu'on les transfere par des changes ou des jeux d'ćcritures au Levant où ce même ducat venitien constitue une monnaie intermationale".

${ }^{42}$ OP. CIT. p. 603: "... Que reçoivent en échange les Barcelonais? Peu de choses, à en croire les archives de leur ville".

${ }^{43}$ C. CARRÉRE, Barcelone, centre économique, II, p. 604; M. DEL TREPPO, Els mercaders, p. 248.

${ }^{4}$ Par exemple, Berenguer de Barcelone (Berengarius de Barcelona, dans les sources ragusaines), fonda en 1341 une société pour le comınerce de la laine et de l'argent en Hongrie. La société a été liquidée en 1341. Deux autres Catalans, Nicolas Anzinelli et Pierre Scolens, tous les deux de Barcelone, reçurent, en 1379, en contrepartie de la marchandise fournie aux Ragusains, 10 livres d'argent et une quantité de marchandises. Cependant, nous
} 
A la différence des Vénitiens, les Catalans n'étaient pas exemptés de l'imposition, lors de l'exportation des métaux précieux de Dubrovnik. Ils devaient en effet remettre une partie de ces métaux a la monnaie de la ville. Aussi, dans le livre du controleur de la monnaie de la ville de Dubrovnik, trouve-on en 1422 le nom du Catalan Bernard Guasquill, procureur de quelques grandes familles marchandes catalanes a Dubrovnik, et lui-même, créditeur des tisserands ragusains, qui vécut à Dubrovnik de 1420 à sa mort, en $1436^{45}$. Au cours de l'année 1422 seulement, Bernard Guasquill avait remis à l'atelier de monnaie ragusain une quantité de deux litres et une once d'argent, et pour cette quantité il a reçu, en contrepartie, 43 hypperperes et neuf gros. Si la quantité d'argent remise par le Catalan aux autorités ragusaines représente $6 \%$ de la quantitée exportée (car tel était selon les dispositions locales le pourcentage à remettre aux autorités pour obtenir un permis d'exportation), il est simple de calculer que Bernard exporta au cours de cette année plus de $11 \mathrm{~kg}$ d'argent $^{46}$. On apprend d'autre part d'une décision du Conseil Mineur de Dubrovnik, datant de 1428, que Bernard Guasquill exportait régulièrement de l'argent et acceptait de payer les impots prévus ${ }^{47}$. La question demeure ouverte, à savoir si les Catalans à Dubrovnik, gens audacieux et entreprenants, respectaient régulièrement l'obligation concernant la remise d'une quantité de métal précieux obligatoire, lors de son exportation de la ville ${ }^{48}$.

Tout dépendait évidemment de l'intérêt pour l'importation des métaux précieux sur les marchés des villes catalanes vers la fin du XIV et le début du XV'e siècle. Depuis qu'en 1346, sous le règne de Pierre IV fut initié la frappe du florin aragonais, à l'instar de la plus célèbre monnaie de même num, frappée en Italie, la frappe de la monnaie d'or dans la Couronne Catalano-Aragonaise était sous le contrôle du pouvoir royal,

ignorons, si ces marchandises sont finalcment parvenues aux ports catalans.

${ }^{45}$ Le registrc du surveillant de la monnaie pour l'année 1422 est le seul qui ait été conservé à Dubrovnik pour tout le XV siècle; il a élé publić par M. DINIC, lz Dubrovackog arhiva II, 1963.

${ }^{46}$ Toute considération plus poussée nous menerait droit au domaine des hypothèses: par exemple, si la quantité évoquéc representait l'exportation annuclle moyenne, Bemard Guasquill, au cours des seize années de scéjour à Dubrovnik, aurait exporté de cette ville plus de $170 \mathrm{~kg}$ d'argent.

${ }^{47}$ Consilium Minus IV, 148'

${ }^{48}$ Nous ne connaissons aucun cas d'amende infligée au marchand catalan pour exportation illégale des métaux précicux. 
tandis que la monnaie d'argent a été laissée sous le contrôle des communautés urbaines ${ }^{49}$. Cette politique du bimétalisme eut pour conséquence les tentatives de l'autorité royale, d'une part, et de l'autorité urbaine, de l'autre, de promouvoir leur monnaie respective au detriment de la monnaie concurente $^{s^{0}}$. La circulation de l'argent dans les villes catalanes était entre les mains des marchands florentins de la Catalogne et du Midi de la France, qui, en ayant imposé un cours suréstimé à ce métal précieux, s'appropriaient d'importantes quantités de l'or, par le biais de l'atelier de monnaie étrangers, toujours par l'intermediaire des marchands florentins. Considérée dans son ensemble, cette politique du bimetallisme, pratiquée dans les pays de la Couronne d'Aragon, favorisait l'importation de l'argent, que cet argent fut changé pour de l'or à Barcelone, ou à Valence, qu'il fut frappé en "croats", ou bien qu'il fut reexporté par les marchands florentins. L'intêrêt pour l'importation de l'argent balkanique dans les pays catalans doit avoir existé, et il pouvait aussi bien interesser les Catalans installés à demeure à Dubrovnik, bien que les preuves positives nous manquent ${ }^{51}$.

Les sources ragusaines laissent supposer qu'outre l'argent, le plomb trouva aussi son chemin vers les marchés des villes catalanes: il est, en effet, bien connu que ce métal fut un des premiers articles d'importation catalans au $\mathrm{XV}^{\mathrm{e}}$ siecle $^{\mathrm{s}}$.

\footnotetext{
${ }^{49}$ F. MATEU Y LiopIS, Glosario hispánico de numisınática, Barcelona 1946.

${ }^{50}$ Le rapport du florin aragonais au croat d'argent a été fixé à $13: 1$, ce qui aboutit à la surévaluation de la inonnaie d'or. Les autorités barcelonaises répondirent à eette inesure par une hausse de la parité de la monnaie d'argent à 8,7:1. Cette parité favorable entraîna l'afflux de l'argent en Catalogne, au cours des premières années du XV siècle. Mais, vers 1425, intervient une chute de l'argent au cours de 10,5:1. Etant donné que le cours officiel de l'or demcura inchangé à 13:1, le tlux s'inversa, et l'argent coınınença à être exporté aux marchés étrangers, surtout en France, ou le rapport de l'or et de l'argent était moins défavorable à ce demier.

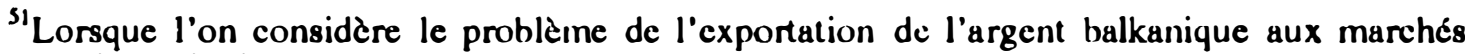
catalans, il est indispensable de rappeler les résultats des recherches de $M$. Del Treppo. Les représentants des grandes maisons de commerce florentines, vendaient à Dubrovnik la laine, que les representants de ces mêmes maisons achetaient en Catalogne. En contrepartie, ils achetaient de l'argent à Dubrovnik. On évoque à ce propos la lettre de Benedetto Cotrugli, à Francesco Nerone, concemant le prix de l'argent à Dubrovnik: cependant, le fait que Francesco Nerone ait développé les affaires à Dubrovnik et à Barcelone en même temps, et qu'il ait acheminé à Dubrovnik la laine catalane, ne signifie pas que l'argent acheté en contrepartie, aboutissait nécéssairement en Catalogne. De Dubrovnik à Venise et Florence, de Florence en France, de France à Barcelone et à Valence. Mais, ce trajet de l'argent des Balkans, pouvait être interrompu à tout moınent dans l'un des ports méditcrranéens, avant l'arrivée en Catalogne.

52 J. VICENS VIVES, Historia econoinica de España, p. 196.
} 
Un certain nombre de marchands catalans, installés à Dubrovnik investissaient d'importantes sommes d'argent dans le commerce du coail ${ }^{53}$. A titre individuel, ou en compagnie avec les patriciens ragusains, ils affretaient les navires et payaient les plongeurs. Il est interessant de signaler que, malgré l'intérêt prononcé pour le corail sur les marchés des villes catalanes, il n' y a pas dans les sources ragusaines de preuve formelle que le corail était acheminé vers la Catalogne, par les soins des Catalans installés a Dubrovnik ${ }^{54}$. Les eaux de Dubrovnik étaient effectivement une région de l'exploitation du corail qui, une fois élaboré, était acheminé vers les riches marchés du Levant: Alexandrie, Beyrouth et Damas ${ }^{55}$. Dans cette activité se signala particulièrement au début du XV siècle la société des Catalans Bernard Guasquill et Jean Ferer et du Ragusain Andre Volcevic. Dans cette société, Bernard affretait les navires et payaient les plongeurs et Jean Ferer, en tant que "socius tractans" vendait le corail au Levant ${ }^{\text {s6. }}$.

Dubrovnik jouait un rôle important en tant que port d'escale pour les navires catalans qui transportaient le bois vers Barcelone et Tortose. De nombreux documents barcelonais témoignent que la ville de Senj, au Nord de l'Adriatique, était, pendant la première moitié du $\mathrm{XV}^{e}$ siècle un important port d' exportation du bois, indispensable aux grands chantiers navals catalans ainsi qu'à la marine marchande ${ }^{57}$. A plusieurs reprises, entre 1430 et 1435, Dubrovnik reçut la visite de Pierre Box, consul catalan à Senj, qui se faisait representer dans la ville par Jean Brull et Bernard Guasquill. Cependant, si l'on juge' d'apres les sources ragusaines, les Catalans

\footnotetext{
${ }^{53} \mathrm{~L}$ 'importance du commerce ragusain des corails a été mis en évidence par I. BozIC, Ekonomski i drustveni razvitak Dubrovnika (Le développement économique et social de Dubrovnik), "Istorijski glasnik", I (1949), pp. 21-61.

${ }^{54}$ Claude Carrère considérait que le corail était un des articles d'exportation vers les marchés adriatiques. Les sources ragusaines témoignent plutôt en faveur de l'exportation du corail vers lcs marchés catalans (Barcelone centre économique, II, p. 601).

${ }^{35}$ B. HRABAK, Ucesce Katalanaca u dubrovackom proinetu zmastom hranom, solju, metalima, koraljima i kreditima do 1520 godine (La participation des Catalans dans le commece ragusain des metaux, du corail, du sel et du crédit, jusqu'en 1520), "Anali zavoda za povjesne znanosti IC JAZU u Dubrovniku", 22-23 (1985), pp. 41-77.

${ }^{56}$ Le prix du corail à Dubrovnik était aux environs de 18 ducats au centenaire.

${ }^{57} \mathrm{C}$. CARRERE, Barcelone centre économique, I, pp. 20, 117, 188; II, 600,601, 602, 603, 606. Les sources catalanes confirment la nomination et le séjour de deux consuls catalans à Senj, Thomas Luis (1418) et Pierre Box (Voir C. CARRERE, op. cit., I, p. 132).
} 
installés à Dubrovnik ne participaient pas régulièrement au commerce du bois entre Senj et les villes catalanes ${ }^{58}$.

Il est légitime de conclure que dans les echanges entre Dubrovnik et la Catalogne, deux articles de commerce jouaient un rôle prépondérant: les esclaves au cours des deux dernières decennies du XIV ${ }^{e}$ siècle et la laine au cours de la première moitie du $X^{\circ}$ siècle. Pour ces deux marchandises seules, on peut affirmer qu'elles traversent tout le chemin qui relie les deux centres éloignés de l'activité économique dans l' Adriatique et la Méditérranée occidentale, qu'elles le traversent sans généralement faire de grandes escales, respectant presque toujours le meme itineraire de la Sicile, avec la seule difference que les esclaves suivent le sens traditionnel d'Orient vers l'Occident, et la laine le sens inverse ${ }^{59}$. La caractéristique commune de ces deux aspects du commerce est de fixer a demeure un certain nombre de marchands de la Catalogne a Dubrovnik.

Les Catalans installés à Dubrovnik participaient au commerce d'un certain noimbre d'articles dont on ne pourrait pas affirmer qu'ils aboutissaient aux marchés de la Catalogne: il s'agissait en premier lieu des métaux précieux, de l'or et de l'argent; ensuite du plomb et du corail. En tant qu'acteurs du commerce maritime mediterranéen, ils fournissaient à Dubrovnik aux individus, aussi bien qu'a la Commune des articles dont on peut pas dire avec certitude qu'ils provenaient de la Catalogne: le ble, le sel, l'huile, le bois, la pierre et divers matériaux de construction.

\footnotetext{
${ }^{58}$ Il est significatif qu'aucun acte pour le trunsport du bois vers les ports catalans n'a été rédigé à Dubrovnik au XV siècle.

${ }^{39} \mathrm{Ceci}$ est confinmé par un grand nombre de contrats concemant la traite des esclaves et le transport de laine rédigés respectiveınent à Duhrovnik et à Barcelone.
} 
TABLFAU A

Liste comparative des prix de lalne sur les marchés catalan et ragusaln au XV slècle

\begin{tabular}{|c|c|c|c|c|c|}
\hline Cote d' archlve ragusaine & Date & Sou: Ducat & $\begin{array}{c}\text { Quantite } \\
\text { Roves=-Milialres }\end{array}$ & $\begin{array}{l}\text { Prlx } \\
\text { Barcelone } \\
\text { Sous=1)ucats }\end{array}$ & $\begin{array}{l}\text { Prix } \\
\text { Dubrovnlk } \\
\text { Ducats }\end{array}$ \\
\hline Div. Not. XIV, 153 & 1. nor: 1427 & $15: 1$ & $11=1$ & $2.31=65,4$ & 58 \\
\hline Div. Not. XVII, 48 & 26 mal 1431 & $15: 1$ & $11=1$ & $176=11,6$ & 60 \\
\hline Div. Not. XVIII,70 & 10 Juillet 1433 & $15: 1$ & $11=1$ & $176=11,6$ & 55 \\
\hline Int. Canc. 11,16 & 20 octobre 1457 & 7 22:1 & $11=1$ & $231=15,4$ & 33 \\
\hline Deb. Not. XXXII,74 & 12 aout 1458 & $22: 1$ & $11=1$ & $231=15,4$ & 32,5 \\
\hline Div. Not. IXI, 184 & 10 dec. 1477 & $24: 1$ & $11=1$ & $231=9,15$ & $40^{1}$ \\
\hline
\end{tabular}

IValeur comparative du florin aragonais par rapport au ducat venitien, exprime en monnale de compte ( sous) au cours du XV sierle

$\begin{array}{lccccc}\text { Annee } & \text { Ducats } & \text { Sous } & \text { Annee } & \text { Flurins aragonals } & \text { Sous } \\ 1+28-1+29 & 1 & 15 & 1426 & 1150 & 13 \\ & & & 1464 & 1 & 14 \\ & & & 1465 & 1 & 15 \\ 1467 & 1 & 22 & 1466 & 1 & 16 \\ 1+71-1472 & 1 & 24 & 1478 & 1 & 17\end{array}$

M. Bloch, tisquisse d' une histoire monetaire de l' Europe, Cahier des annales $9,1954$.

R. Lopez, Scttecento anni fa, il ritorno all' oro nell' occidente duecentesco, Rivista storica Italiana,65-66

c. Cippola, Monetia e clvilta mediterranea, Venezia 1957

F. Mateu y Llopis, Glossarium numismaticum Hispaniae, Barcelona 1946;

TABLEAU B

Les cargalsons de lalne embarquées dans les ports catalans en destInatlon de Dubrovnlk

\begin{tabular}{|c|c|c|c|}
\hline Cote & Date & Quantlté & Prlx \\
\hline B. Masons, $121^{\prime}$ & 6 septembre 1428 & 458 roves, 22 llvres $=4772 \mathrm{~kg}$ & 450 llvres \\
\hline B. Masons, 23'-24 & 6 septembre 1428 & 393 quintaux, 2 roves $=16369,6 \mathrm{~kg}$ & 1600 llvres \\
\hline B. Masons, 34 & 9 octobre 1428 & 243 roves, 15 llvres $=2527 \mathrm{~kg}$ & 200 llvres \\
\hline R. Masons, 94'-95 & 21 Juln 1429 & 515 quintaux, 1 rove, 1 llvre $=21684 \mathrm{~kg}$ & 2000 Ilvres \\
\hline 8. Masons, 119 & 5 sept. 1429 & $341 / 2$ quIntaux $=1435 \mathrm{~kg}$ & 155 llvres 1 \\
\hline
\end{tabular}

${ }^{\prime} D$ ' après A.ll.P. B. Bartholome Masons, Lbros de seguros maritimos ( 2 julio 14 $28-23$ diclembre 1429) 


\section{RÉSUMÉ}

Bien que séparées par des centaines de milles d'espace maritime, les villes de la côte catalane et Dubrovnik entretenaient, à la fin du Moyen Age, d'intenses rapports économiques. Un grand nombre de Catalans se deplaçaient de Barcelone, Tortose, Tarragone et d'autres villes, principalement de la côte méditerranéenne, mais aussi de l'arrière-pays, pour aller s'installer dans la ville de Dubrovnik, sur la côte adriatique. Cet article se propose d'étudier d'une part les échanges économiques entre les villes catalanes et Dubrovnik et, d'autre part, de reconstituer le destin des familles catalanes et des individus, qui choisissaient de s'expatrier dans cette ville slave, loin de leur pays et de leur ville d'origine. L'auteur s'appuie sur les documents des archives catalanes, principalement des Archives de la Couronne d'Aragon et des Archives Notariales de Barcelone, ainsi que des Archives de Dubrovnik, pour presenter l'exportation catalane à Dubrovnik (laine) et l'exportation ragusaine dans les villes de Catalogne (esclaves, métaux precieux, bois, etc.). On suit aussi le sort des Catalans à Dubrovnik (conflits, adaptation, position dans la société locale). La nature des documents notariaux lui permet de proceder à certaines évaluations statistiques, sur la nature et le rythme des échanges.

\section{SUMMARY}

Although the distance between the cities of the catalan coast and Dubrovnik is several hundred miles, these cities maintained, during the last centuries of the Middle Ages, intense economic relations. A great number of Catalans were leaving Barcelona, Tortosa, Tarragona and other important towns in the first place of the coast but also in the hinterland, in order to settle in Dubrovnik on the adriatic coast. This article deals, firstly, with the economic exchanges between the catalan towns and Dubrovnik, but also with individual destinies of people from Catalonia who chose to live in the slavic town of Dubrovnik, far away from their homeland. The author has investigated essentially in the Archives of the Crown of Aragon, in Barcelona, and in the Notarial Archives of the same city, as well as in the Archives of Dubrovnik, in order to study the export of catalonian products to Dubrovnik (wool, in the first place) and the import from Dubrovnik to 
Catalonia (slaves, precious metal, timber, etc.). He also deals with lives of Catalans in Dubrovnik (their integration, their conflicts, etc.). The study is based on documents which allow good statistical evaluations on the nature and the tempo of exchanges. 